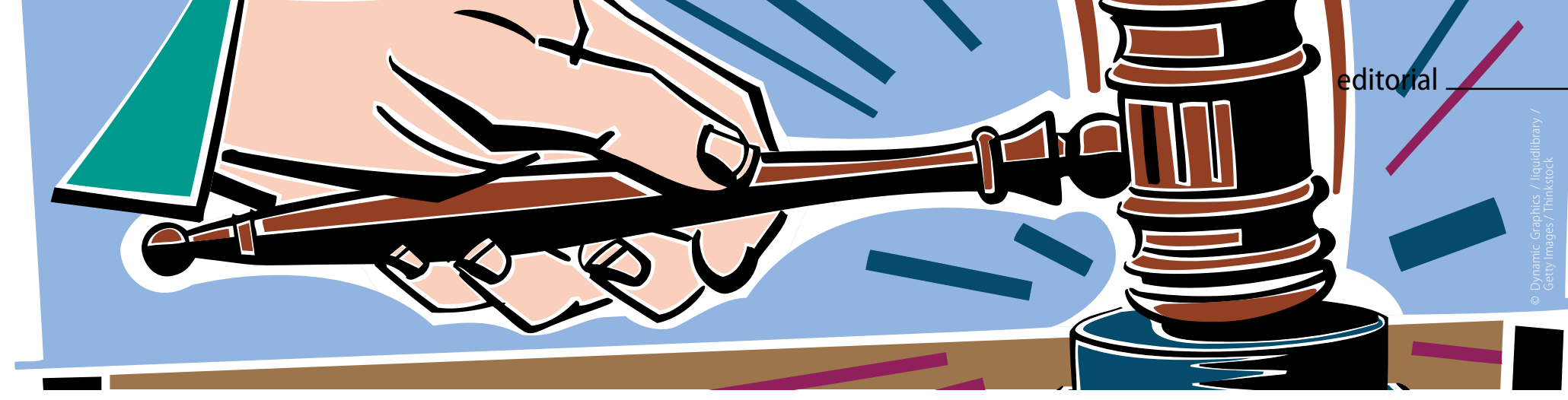

\title{
Eine Therapie mit verpflichtender Evidenz
}

Die Effizienz einer komplexen, multimodalen pneumologischen Rehabilitation (PR) gilt als bewiesen. Wäre PR ein Medikament, so wäre die ausbleibende Verordnung als Kunstfehler anzusehen. Dies gilt besonders für die COPD. Neuere Daten belegen, dass auch Patienten, die an Asthma bronchiale oder an interstitiellen Lungenerkrankungen leiden, durch eine PR hinsichtlich Lebensqualität und Leistungsfähigkeit profitieren. Für die COPD gibt es sogar Hinweise, dass durch eine PR die Mortalität gesenkt werden kann. In jedem Fall sinkt die Zahl notwendiger Rehospitalisationen nach akuter Exazerbation (AECOPD). Nach dieser Evidenz richtet sich die Initiative „choosing wisely“ im Deutschen Ärzteblatt 5/16, in der eine PR nach AECOPD als eine von fünf sinnvollen Maßnahmen empfohlen wird.

Wenn nun eine derart „verpflichtende Evidenz“ vorliegt, stellt sich die Frage, wieso die Inanspruchnahme in Deutschland so gering ist? Exakte Zahlenangaben hierzu fehlen. Es wird vermutet, dass nur 4 bis 5\% derer, die wegen AE-COPD stationär behandelt wurden, im Anschluss eine PR erhalten. Hierfür sind meines Erachtens vier Ursachen zu diskutieren.

Erstens der Patient selbst: Nur 9\% der potenziellen Kandidaten beendeten eine PR in zwei britischen Studien. Teils lehnen sie eine PR ab oder meinen, die COPD ausreichend unter Kontrolle zu haben. Bei anderen Patienten fehlt die Krankheitseinsicht (low compliance) oder sie scheuen eine PR wegen Angst/ Depression. Ob dies auch für Deutschland gilt, wo die stationäre PR vorherrscht, ist unklar. Ich glaube, dass vielen Fantasie und Mut fehlen, gegen ihre krankheitsbedingten Einschränkungen anzukämpfen. Mitunter sind es frustrane Vorerfahrungen, die eine erneute PR für Patienten nicht sinnvoll erscheinen lässt.

Die zweite Ursache sind die Kostenträger: Für sie stellt eine PR in unserem Gesundheitswesen eine der wenigen Möglichkeiten dar, Ausgaben durch Ablehnung zu verhindern. Speziell bei Patienten mit weit fortgeschrittenen Lungenerkrankungen sehen Krankenkassen bzw. MDK (teils wegen Unwissenheit?) kein Reha-Potenzial mehr. Diese Ansicht ist eher den traditionellen Vorstellungen der Leistungsfähigkeit einer PR geschuldet. Bei guter apparativer, personeller Ausstattung und fachlicher Expertise ist eine multimodale, multidisziplinäre Rehabilitation jedoch in der Lage, die oft große Lücke zwischen klassischer Rehabilitation und Akutversorgung $\mathrm{zu}$ schließen. Studien zeigen, dass gerade Patienten mit stärksten Einschränkungen am meisten von einer komplexen PR profitieren.

Durchaus verständlich ist auch, dass sich viele Niedergelassene und Klinikärzte, die PR verordnen, auf dem bürokratischen Weg von Antragstellung, Ablehnung, Widerspruch usw. resig-

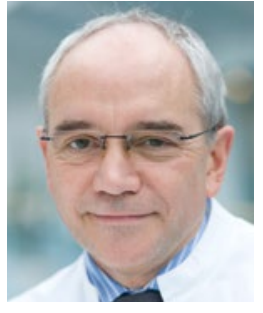

Prof. Dr. med. Klaus Kenn

Chefarzt Schön Klinik Berchtesgadener Land Malterhöh 1, 83471 Schönau am Königssee KKenn@Schoen-Kliniken.de

niert nicht mehr in der Lage sehen, den mühsamen Kampf im Einzelfall zu Ende zu führen. Hinzukommt, dass leider wegen erheblicher Qualitätsunterschiede angebotener PR viele Ärzte von deren Ergebnissen wenig überzeugt wurden, sodass ihr Enthusiasmus, eine PR weiter zu verordnen, limitiert ist.

Der vierte Grund sind die PR-Anbieter: In der Realität ist PR kein wirklich geschützter Begriff. Zwar existieren verschiedene Vorgaben, die aber nicht wirklich überprüft werden. Somit ist das Angebot, was sich hinter dem Begriff PR verbirgt, sehr breit gefächert und reicht von „niederschwelligen“ bis hin zu hoch differenzierten, komplexen Therapiemaßnahmen. Wenn außen „Reha“ drauf steht, muss auch innen wirkliche „Reha“ drin sein. In der Schweiz werden Zertifizierungsverfahren für PR-Anbieter durchgeführt. Dies erscheint mir auch für unser Gesundheitssystem empfehlenswert. Weltweit wird PR dominierend ambulant angeboten, in Deutschland existiert diese Option eigentlich nicht. Daher ist es nötig - dies sage ich als Vertreter stationärer PR - dringend ambulante PR-Angebote zu schaffen. Anderenfalls ist die „choosing wisely“-Empfehlung gar nicht umzusetzen. Man sollte die Chance nutzen, primär eine ausreichend inhaltliche Expertise einzufordern, um zu verhindern, dass die „Umetikettierung“ der Rehaeinrichtung echte pneumologische Expertise ersetzt. Der stationären PR bleiben mit der Versorgung komplexer Kranker genügend Aufgabenfelder.

Oberstes Ziel der PR ist nicht die kurzfristige Verbesserung von Leistungsfähigkeit und/oder Lebensqualität. Die wirkliche Herausforderung stellt die Verhaltensänderung hin zu einer nachhaltig gesünderen Lebensform dar. Der körperlichen Aktivität kommt dabei eine herausragende Bedeutung zu. An diese muss der Patient zwar durch eine PR herangeführt werden, die wiederholte Motivation zur Beibehaltung eines erhöhten Aktivitätsniveaus stellt meiner Ansicht nach aber eine gemeinsame Aufgabe für alle am Behandlungsprozess Beteiligte dar. 